\title{
771 NOVEL LIPID NANOPARTICLE VACCINE PLATFORM FOR EFFICIENT DELIVERY OF HIGH- AND LOW-AFFINITY EPITOPES
}

'Unnur Jóna Björgvinsdóttir*, '2Laura Stentoft Carstensen*, 'Anna Colliander, 'Ditte Elisabeth Jæhyer, ${ }^{1}$ Gael Clergeaud Veiga, ${ }^{1}$ Hólmfríður Rósa Halldórsdóttir, ${ }^{1}$ Matilde Smærup Jørgensen, ${ }^{1}$ Esben Christensen, ${ }^{1}$ Sara Vangsgaard, ${ }^{1}$ Aristeidis Koukos, ${ }^{1}$ Martin Bak, ${ }^{1}$ Paul Kempen, 'Thomas Lars Andresen. 'Technical University of Denmark, Lyngby, Denmark; ${ }^{2}$ Technical Univeristy of Denmark, Lyngby, Denmark

Background Therapeutic cancer vaccines represent an intriguing approach to cancer immunotherapy and they have been widely explored for the last decade. As opposed to standard modalities, such as surgery and chemotherapy, an effective vaccine-based immune response may provide protection against metastatic disease. Peptide based vaccines can elicit a highly targeted immune response and include a simple, fast and costeffective production due to recent developments in solid phase peptide synthesis. Recent development within the field of COVID-19 vaccines has highlighted the use of lipid nanoparticles as an effective drug delivery system for vaccination. Incorporation of peptide antigens into engineered micro- and nanoparticles enables induction of a potent $\mathrm{T}$ cell response, partly attributed to prolonged and improved antigen presentation by dendritic cells after particle internalization. Peptidebased vaccines are often based on delivery of high-affinity $\mathrm{T}$ cell model epitopes. However, the therapeutic relevance of vaccination with low-affinity epitopes is gaining increasing support following the observation that high-affinity epitopes can promote $\mathrm{T}$ cell exhaustion resulting from excessive $\mathrm{T}$ cell receptor stimulation. Here, we characterize and evaluate a novel lipid nanoparticle (LNP) vaccine platform that is suited for delivery of both high- and low-affinity epitopes in the setting of therapeutic cancer vaccination.

Methods LNPs were formulated to carry high- or low-affinity peptide epitopes from Ovalbumin (OVA) in conjunction with the TLR7 agonist 1V270. The peptides were anchored to the surface of the LNPs via a reducible DSPE-PEG2000 linker system. The therapeutic vaccine platform was evaluated in vivo both as a monotherapy and in combination with adoptive transfer of OT-I T cells in the syngeneic B16-OVA murine melanoma model.

Results The LNP vaccine promotes efficient antigen-release and ensures high, continuous antigen-presentation by antigenpresenting cells. While the LNPs can be administered via multiple routes, intratumoral vaccination favors enhanced particle uptake in dendritic cells in the tumor. Formulated with either high- or low-affinity epitopes, intratumorally delivered vaccine particles promote superior tumor-infiltration of adoptively transferred $\mathrm{T}$ cells, which translates into potent anti-tumor efficacy in vivo. Finally, we show that vaccination with both $\mathrm{CD} 8+$ and CD4+ epitopes can delay tumor growth and prolong survival in an antigen-dependent manner.

Conclusions This study presents a versatile and multi-purpose LNP vaccine platform that ensures effective delivery of highand low-affinity epitopes. Intratumoral administration promotes vaccine particle uptake by intratumoral dendritic cells, which is followed by $\mathrm{T}$ cell infiltration and anti-tumor efficacy in vivo.

Ethics Approval All animal procedures were approved by the Danish National Animal Experiments Inspectorate. 